\title{
DOS ESQUEMAS DE COMISIONES A LAS AFP EN EL PERÚ. ¿CUÁL CONVIENE?
}

\author{
TWO SCHEMESTO AFP COMMISSIONS IN PERU. ¿WHICH IS? \\ Esteban Marino Avelino Sánchez \\ Héctor Félix Cerna Maguiña ** \\ Docentes Asociados de la Facultad de Ciencias Contables \\ Jaime Bernabé Torres Ramírez *** \\ Docente Auxiliar de la Facultad de Ciencias Contables \\ Universidad Nacional Mayor de San Marcos-UNMSM / Lima-Perú \\ [Recepción: Mayo de 2013/ Conformidad: Junio de 2013]
}

\section{RESUMEN}

El problema de muchos afiliados al sistema privado de pensiones del Perú, en estos días, es la elección del esquema de comisiones a las AFP; la Ley 29903 establece dos: un porcentaje sobre el sueldo, tal como se viene haciendo o una comisión mixta, conformada con un porcentaje decreciente sobre el sueldo y otro sobre el saldo acumulado, durante 10 años; si los afiliados no eligen hasta el 31 de mayo el porcentaje sobre el sueldo, automáticamente la mayoría pasarán al esquema de la comisión mixta, advierten los expertos. De acuerdo con nuestras estimaciones efectuadas en el numeral 3, la diferencia entre ambos esquemas sería mínima, imperceptible para la mayoría de afiliados, porque las AFP tienen libertad para subir o bajar la comisión anual sobre el saldo acumulado a su voluntad; la opción de los afiliados por uno u otro esquema no afecta la libertad de las AFP para modificar la comisión anual, de acuerdo a las señales del mercado.

Palabras clave: esquemas de comisiones, a las AFP, Ley 29903, fondos de pensiones en el SPP, tipo 0.

\begin{abstract}
The problem with many members of the private system of pension in Peru, these days, is the election commission scheme to AFP, Law 29903 establishes two: a salary percentage, as is being done or a joint committee, formed with a decreasing percentage of the salary and another on the accumulated balance for 10 years, if the members does not choose until May 31th, the salary percentage, automatically most of them will pass to the mix plan of commission, experts warn. According to our estimates made in Section 3, the difference between the two plans would be minimal, imperceptible to most of the members because the AFP are free to raise or lower the annual fee on the accumulated balance to his will, the choice of members by one or another plan does not affect the freedom of AFP to modify the annual fee, according to market signals.
\end{abstract}

Keywords: plans of commissions, AFP, Law 29903 pension funds in the SPP, type 0.

\footnotetext{
* Magíster en Contabilidad con Mención en Auditoría, Contador Público y Economista Colegiado, Perito Contable de la Corte Superior de Justicia de Lima.

** Magíster en Docencia Universitaria, Lic. Investigación Operativa, Colegiado en el COMAP.

*** Maestrista en Docencia Universitaria y en Geofísica, Lic. Investigación Operativa, Colegiado en el COMAP.
} 


\section{INTRODUCCIÓN}

En este artículo damos un vistazo, desde el lado de aportes y comisiones, al sistema privado de pensiones del Perú desde su creación a la fecha, luego presentamos nuestros comentarios sobre la Ley 29903 y disposiciones reglamentarias de la Superintendencia de Banca, Seguros y AFP (SBS) y un ejemplo de cálculo para el primer periodo bienal, con los dos esquemas de comisiones: sobre el flujo y mixta (con sus dos componentes) establecidos en la Ley 29903.

Cabe recordar, de las ocho AFP que iniciaron operaciones en 1993, dos dejaron de operar antes de un año, otra siguió el mismo camino en setiembre de 1996, dos se fusionaron en enero del 2000; en agosto de 2005 ingresa al mercado de administración de fondos de pensiones una nueva AFP, un año después esta absorbe a las AFP fusionadas en el año 2000. Del año 2000 al 2012 operaron, prácticamente, cuatro AFP; a partir de abril de 2013, con el ingreso de Hábitat serán cinco, podrían bajar levemente las comisiones. El aporte al fondo de pensiones hasta julio de 1995 fue de $10 \%$ de la remuneración asegurable, por Ley 26504 y prórrogas sucesivas, a partir de agosto de 1995 hasta diciembre de 2005 disminuyó a 8\%; a partir de enero de 2006 se restableció lo dispuesto en el texto original del Decreto Ley 25897: 10\% de la remuneración asegurable.

Hasta el año 2005, los expertos señalan al sistema privado de pensiones del Perú, con una tasa promedio de $2.26 \%$ sobre la remuneración (año 2003), como el más oneroso de América Latina. En relación con el aporte al fondo de pensiones, la comisión mensual de las AFP representó el 28.25\% de rentabilidad (2.26\% entre $8 \%$, hasta 2005 ). En la última década la rentabilidad de las AFP estaría bordeando el $20 \%$ en relación al aporte mensual al fondo.

Con la reciente reforma del sistema privado de pensiones, las AFP pueden cobrar sus comisiones de dos formas: un porcentaje del sueldo, tal como viene sucediendo desde 1993, o una combinación, un porcentaje del salario y otro del saldo acumulado, al que se llama mixto; durará 10 años y luego de ello solo incluirá la comisión sobre el saldo. No me queda claro qué pasaría con el otro esquema en el futuro. Los afiliados tuvieron tiempo hasta el 31 de mayo de 2013 para decir si permanecen en el esquema conocido o migrar al esquema mixto, la decisión habría sido complicada por la abundancia de información de los expertos.

De acuerdo con nuestras estimaciones cuantitativas, la diferencia entre ambos esquemas sería mínima, imperceptible para la mayoría de afiliados; pues las AFP tienen libertad para subir o bajar la comisión anual sobre el saldo acumulado a su voluntad, la opción de los afiliados por cualquiera de los esquemas no afecta la libertad de las AFP para modificar la comisión anual, de acuerdo a las señales del mercado.

\section{ANTECEDENTES}

Mediante el Decreto Ley 25897, del 6 de diciembre de 1992, se creó el Sistema Privado de Pensiones del Perú, modificado varias veces; por ello, conforme lo dispone el Decreto Legislativo 874 de noviembre de 1996, se elaboró un texto único ordenado (TUO), el que fue publicado en abril de 1997, vigente con algunas modificaciones.

Los aportes del trabajador, según el texto original del Decreto Ley 25897 (artículo 30) fueron: al fondo de pensiones $10 \%$ de la remuneración asegurable -RA, prima de seguro un porcentaje de la RA, contribución de solidaridad $1 \%$ de la RA, comisión variable de la AFP un porcentaje de la RA y comisión fija de la AFP en unidades monetarias, sin precisar el importe. Cabe señalar, de las ocho AFP que iniciaron sus operaciones en 1993, dos dejaron de operar antes de un año, otra en setiembre de 1996, dos se fusionaron en enero del 2000. En agosto de 2005 una nueva AFP ingresó al mercado de administración de fondos de pensiones, la que un año después absorbió a la AFP Unión Vida. A partir del año 2000, prácticamente, cuatro AFP operan en el mercado de administración de fondos de pensiones.

Las comisiones de las AFP en el Perú hasta diciembre de 2005: 2.26\% sobre la remuneración, según sostiene Eduardo Morón en su libro electrónico: "Diez años del Sistema Privado de Pensiones"

42/ QVIPURAMAYOC | Vol. 21(39) 2013 
(2003; 94) fueron las más onerosas de América Latina; pues el porcentaje de $2.26 \%$ representó el $28.25 \%$ de rentabilidad $(2.26 \%$ entre $8 \%$ al fondo de pensiones). A partir de enero de 2006 con la tasa del texto original: $10 \%$ de aportes al fondo de pensiones, dicha rentabilidad, sin duda, se ha reducido; pero todavía sería alta para compensar el tamaño reducido del fondo de pensiones peruano, equivalente a la octava parte de su similar chileno.

Cabe señalar, también, la investigación: "Cálculo de Reservas Actuariales en los Sistemas de Pensiones Administradas por el Estado", del Profesor Héctor Cerna y otros, el que contribuye al estudio de los sistemas de pensiones del Perú.

\section{DISPOSICIONES DE LA LEY 29903}

La Ley 29903, de julio de 2012, modificó el TUO del sistema privado de pensiones del Perú y adicionó nuevos textos; sobre modalidades de comisiones a las AFP, incorporación de nuevos afiliados y cuatro tipos de fondos de pensiones. La Ley 29903 establece dos modalidades de comisiones a las AFP (excluyentes en los próximos 10 años): un porcentaje sobre la remuneración mensual, que ya vienen cobrando las AFP desde 1993, y la comisión mixta (un porcentaje sobre el saldo acumulado y otro decreciente sobre la remuneración mensual); así como la obligación de las AFP, en el caso de fondos obligatorios, de administrar cuatro tipos de fondos de pensiones.

Los nuevos afiliados que se incorporen al sistema privado de pensiones, de acuerdo con los artículos 6, 7, 7-A y 24 -inciso d, se afiliarán a la AFP adjudicataria que ofrezca cobrar la menor comisión mixta; conformada por dos componentes: una comisión porcentual sobre la remuneración mensual del afiliado y una comisión sobre el saldo del fondo de pensiones administrado por los nuevos aportes que se genere a partir de la entrada en vigencia de la primera licitación a la que se refiere el artículo 7-A. La SBS, con la opinión favorable del MEF, establecerá las condiciones para la aplicación de la comisión sobre la remuneración asegurable -RA por un plazo determinado, sobre la base de una trayectoria decreciente en el tiempo. La comisión sobre el saldo por los nuevos aportes será fijada libremente por las AFP. Una vez agotado el plazo de 10 años, solo se aplicará la comisión sobre el saldo.

Para los afiliados existentes se aplicará una comisión mixta sobre sus nuevos aportes, en los plazos y medios que establezca la SBS, salvo que manifiesten su decisión de permanecer en la modalidad de la comisión porcentual sobre la remuneración asegurable. La SBS podrá establecer mecanismos y condiciones de licitación de diferente naturaleza con la finalidad de promover la competencia en el mercado; en cualquier caso, la SBS dictará las normas reglamentarias sobre la materia.

Tratándose de aportes obligatorios, dice el artículo $18^{\circ}$-A, las AFP administrarán obligatoriamente cuatro tipos de fondos de pensiones: tipo 0 , tipo 1 , tipo 2 y tipo 3 . El fondo de pensiones tipo 0 , se orienta a mantener el valor del patrimonio de los afiliados con crecimiento estable y muy baja volatilidad; es de carácter obligatorio para los fondos de todos los afiliados de 65 años cumplidos hasta que opten por una pensión de jubilación, salvo que el afiliado exprese por escrito su voluntad de asignar su fondo al tipo 1 o al tipo 2. El fondo de pensiones tipo 1 , se orienta al crecimiento estable del patrimonio de los afiliados con baja volatilidad; este tipo de fondo es de carácter obligatorio para los fondos de todos los afiliados mayores de 60 años y menores de 65 años. El fondo de pensiones tipo 2, se orienta al crecimiento moderado del patrimonio de los afiliados con volatilidad media. El fondo de pensiones tipo 3, se orienta a un alto nivel de crecimiento del patrimonio de los afiliados con alta volatilidad.

\section{DISPOSICIONES DE LA RESOLUCIÓN SBS No 8514-2012}

La resolución indicada define los conceptos vinculados a las dos modalidades de comisiones de las AFP, establece el cronograma y el procedimiento para calcular el factor (porcentaje) decreciente, el que se aplicará sobre la remuneración mensual del afiliado que haya elegido la comisión mixta, para retribuir a la AFP por la administración de su fondo de pensiones, como puede observar en el cuadro 1 . 


\section{Cuadro 1}

Cronograma de aplicación de los factores a la comisión base sobre el flujo

\begin{tabular}{|c|c|}
\hline Año & Factor \\
\hline Febrero 2013 - Enero 2015 & $86,5 \%$ \\
\hline Febrero 2015 - Enero 2017 & $68,5 \%$ \\
\hline Febrero 2017 - Enero 2019 & $50,0 \%$ \\
\hline Febrero 2019 - Enero 2021 & $31,5 \%$ \\
\hline Febrero 2021 - Enero 2023 & $13,5 \%$ \\
\hline Febrero 2023 en adelante & $0 \%$ \\
\hline
\end{tabular}

La comisión base para el cálculo de la trayectoria (factor) decreciente -cada dos años-, es la comisión registrada al mes de agosto de 2012 en las AFP existentes. Tratándose de una nueva AFP será $1.75 \%$, dicen los artículos 3-6 de resolución bajo comentario. La comisión sobre el saldo se aplicará sobre los aportes acumulados en la CIC, que se constituyan a partir de la vigencia de la primera licitación aludida en el artículo 7-A.

La comisión mixta se aplicará respecto de los nuevos aportes que se devenguen a partir del día siguiente de la fecha de término para el ejercicio de la opción de elección del afiliado (31 de mayo de 2013), dicen los artículos 7, 8 y 9 de la resolución bajo comentario.

Para que el afiliado pueda efectuar la elección, de modo informado, la modalidad de comisión que le conviene pagar, las AFP deberán identificar la base de datos de sus afiliados, a quienes les enviarán una comunicación escrita, por medios físicos o electrónicos, a criterio de la AFP, informándole los aspectos detallados en los acápites i-viii, dice el artículo 10 de dicha Resolución (entre otros, si el afiliado no elige la modalidad de comisión que le conviene pagar, en el plazo establecido, sus aportes se sujetarán al esquema mixto). Así mismo, las AFP deberán realizar una campaña informativa y de orientación que permita tomar conocimiento oportuno y adecuado a los afiliados al sistema privado de pensiones sobre la posibilidad de permanecer en el esquema de cobro de la comisión sobre flujo o pasar a la comisión mixta, proveyendo información de cada uno de los esquemas, los plazos y medios para realizar la elección. La opción por la comisión sobre flujo (sobre la remuneración mensual) permite a los afiliados revocarla una sola vez, en el plazo de 6 meses contados desde la fecha de término para la elección de la opción.

\section{ELEGIREL ESQUEMA DE PAGO HABRÍA SIDO UNA DECISIÓN COMPLICADA}

La SBS, con la finalidad de facilitar a los afiliados al sistema privado de pensiones a tomar una mejor decisión sobre el esquema de comisiones que les conviene, en su página web -dice Javier Olivera en la dirección electrónica indicada en la bibliografíacolgó una calculadora, la base de supuestos e información puntual con la cual el afiliado podía hacer hasta 3,384 combinaciones posibles, lo que revela una decisión muy complicada, porque depende de muchas variables; más todavía cuando se desconoce al término de 10 años, el futuro de uno y otro esquema. De ahí que, al término de la segunda prórroga (31 de mayo de 2013), menos de la tercera de afiliados a las AFP decidió permanecer en el sistema conocido; mientras que 3.8 millones habrían pasado por omisión o voluntariamente al esquema de la comisión mixta.

Otro objetivo de la reforma del sistema privado de pensiones de 2012 habría sido, para forzar la competencia entre las actuales AFP peruanas y dos potenciales entrantes, la primera subasta de afiliación en los próximos dos años (alrededor de $700 \mathrm{mil}$ trabajadores), en la que salió ganadora Hábitat AFP. ¿Por qué se hizo de esta manera?, pregunta Eduardo Morón y contesta: "simplemente porque los afiliados, a pesar de observar comisiones menores o rentabilidades mayores, no se trasladaban. Las razones 
de esta inacción son en parte que la gente no entiende el sistema, que ofrece un beneficio (dentro) de 40 años a nadie le interesa sino cuando falta muy poco" (Blog/Economía sin anestesia, 21 dic, 2012).

\section{LA DIFERENCIA EN EL PRIMER PERIO- DO BIENAL ES IMPERCEPTIBLE}

\section{Comisiones en el primer periodo bienal.}

Considere, al trabajador $\mathrm{xx}$ con remuneración mensual de S/. 3 500.00, afiliado a la AFP Profuturo, la cual tiene la comisión más alta: $2.10 \%$ de marzo de 2013 y $2.10 \%$ de agosto de 2012 (vea cuadro 2).

a) Si el trabajador xx permanece en el esquema conocido (comisión sobre la remuneración), su remuneración mensual S/. 3500.00 y la comisión de $2.10 \%$, igualmente, se mantienen sin variación los dos años siguientes; la comisión mensual a la AFP es S/. $73.50(3500 \times 0.0210)$ al final de cada mes. $\mathrm{Su}$ valor actual con la tasa de $12 \%$ efectiva anual (0.00948879 TEM) y 24 periodos mensuales resulta $S / .1570 .93(73.50 \times 21.3732267)$.

b) Si el trabajador xx cambia de esquema a la comisión mixta, que tiene dos componentes: 1) la comisión decreciente sobre el flujo y 2) un porcentaje sobre el saldo acumulado del fondo; con la misma remuneración la comisión decreciente sobre el flujo con la tasa $1.85 \%$ (vea cuadro 3) es S/. 64.75 (3 500 x 0.0185 ) al final de cada mes. Su valor actual con la misma tasa de $12 \%$ efectiva anual (0.00948879 TEM) y 24 periodos mensuales resulta S/. 1383.92 (64.75 x 21.3732267).

La diferencia S/. 187.01 (1 570.93 - 1383.92 ) representa menos del $12 \%$ de la comisión del esquema conocido (comisión sobre la remuneración

\section{Cuadro 2}

Comisiones de las AFP y Prima de Seguro

(\% de la remuneración asegurable)

\begin{tabular}{|l|c|c|c|c|c|c|}
\hline \multirow{3}{*}{ AFP } & \multicolumn{2}{|c|}{ Agosto de 2012 } & \multicolumn{4}{c|}{ Marzo de 2013 } \\
\cline { 2 - 6 } & $\begin{array}{c}\text { Comisión } \\
\text { sobre flujo }\end{array}$ & $\begin{array}{c}\text { Prima de } \\
\text { seguro }\end{array}$ & \multirow{2}{*}{$\begin{array}{c}\text { Comisión } \\
\text { sobre flujo }\end{array}$} & \multicolumn{2}{c|}{ Comisión mixta } & Prima de \\
\cline { 5 - 6 } & & CSF $\left(^{*}\right)$ & CASS $(+)$ & seguro \\
\hline Horizonte & 1.95 & 1.36 & 1.85 & & & 1.38 \\
\hline Integra & 1.80 & 1.16 & 1.74 & & & 1.22 \\
\hline Prima & 1.75 & 1.29 & 1.60 & 1.51 & 1.90 & 1.30 \\
\hline Profuturo & 2.14 & 1.42 & 2.10 & & & 1.41 \\
\hline
\end{tabular}

(*) CSF: Comisión sobre flujo. (+) Comisión anual sobre el saldo (\% sobre el saldo acumulado) Fuente: Portal electrónico de la SBS.

mensual), la que puede ser cubierta con la comisión anual sobre el saldo, determinada libremente por la AFP. En general, la diferencia por la participación o no del afiliado en la elección del esquema (la comisión sobre el flujo o sobre el saldo) sería mínima, imperceptible para la mayoría de afiliados; pues las AFP tienen libertad para subir o bajar la comisión anual sobre el saldo acumulado a su voluntad.
La comisión anual sobre el saldo (CASS) es un componente del esquema mixto. El cálculo de la reducción de la comisión, en porcentajes, sobre el flujo para dos AFP: una existente con comisión base de 2.14\% (agosto de 2012), la más alta del mercado y una nueva AFP con porcentaje de $1.75 \%$ se efectúa multiplicando el factor de la tabla 2 por cada una de las tasas antes mencionadas, los resultados se presentan en el cuadro 3 . 


\section{Cuadro 3}

Factores para la reducción de comisiones sobre el flujo

\begin{tabular}{|c|c|c|c|}
\hline Año & Factor & De AFP antigua & De AFP nueva \\
\hline Febrero 2013 - Enero 2015 & $86,5 \%$ & $1.85 \%$ & $1.51 \%$ \\
\hline Febrero 2015 - Enero 2017 & $68,5 \%$ & $1.47 \%$ & $1.20 \%$ \\
\hline Febrero 2017 - Enero 2019 & $50,0 \%$ & $1.07 \%$ & $0.88 \%$ \\
\hline Febrero 2019 - Enero 2021 & $31,5 \%$ & $0.67 \%$ & $0.55 \%$ \\
\hline Febrero 2021 - Enero 2023 & $13,5 \%$ & $0.29 \%$ & $0.24 \%$ \\
\hline Febrero 2023 en adelante & $0 \%$ & $0.00 \%$ & $0.00 \%$ \\
\hline
\end{tabular}

\section{CONCLUSIONES}

1. En general, la diferencia por la participación o no del afiliado en la elección de uno u otro esquema sería mínima, imperceptible para la mayoría de afiliados; las AFP tienen libertad para subir o bajar la comisión anual sobre el saldo acumulado a su voluntad, la opción de los afiliados por uno u otro esquema no afecta la libertad de las AFP para modificar la comisión anual, de acuerdo a las señales del mercado.

2. En el experimento efectuado, para el primer periodo bienal, se utiliza la comisión más alta del mercado; con comisiones menores, la diferencia porcentual sería menor, quizá menos del 10\%.

3. Los afiliados al sistema privado de pensiones de Perú, evidencian alta fidelidad con su AFP elegida o no, a pesar de observar comisiones menores o rentabilidades mayores, no se trasladaban a otra AFP, quizá porque desconocen las bondades del sistema.

4. La dispersión de normas del sistema privado de pensiones, como: artículo 7-A, artículo 7-B, artículo 7-C, artículo 7-D, artículo 7-E, artículo 7-F y las modificaciones parcial o total de otros artículos, dificultan una apreciación apropiada de las bondades del sistema privado de pensiones del Perú; se requiere un nuevo texto único ordenado.

5. Al vencimiento del plazo para decidir sobre el esquema de comisión a la AFP, el 31 de mayo de 2013, menos de la tercera de afiliados a las AFP permanecerían en el sistema conocido; mientras que 3.8 millones habrían pasado por omisión o voluntariamente al esquema de la comisión mixta.

\section{REFERENCIAS BIBLIOGRÁFICAS}

1. Gobierno del Perú. 1992 Decreto Ley 25987, Ley del Sistema Privado de Pensiones y modificatorias. 2012 LEY No 29903 y Decreto Supremo N¹37-2012-EF.Fecha de publicación: 19 de julio y 05 de agosto de 2012, respectivamente.

2. Superintendencia de banca, Seguros y Administradoras Privadas de Fondos de Pensiones 2012 Resolución SBS No 8514-2012. Normas para la Reducción y Elección de la Comisión sobre el Flujo - Ley 29903 y Circular No AFP -1272012. Lineamientos para la aplicación de comisiones sobre el saldo administrado en el caso de los aportes obligatorios. Vigentes a partir del: 09/11/12.

3. Esteban Avelino. 2001 Legislación Laboral y de Seguridad Social. Editorial San Marcos. Lima - Perú.

4. Morón Eduardo, y Eliana Carranza (2003). Diez años del Sistema Privado de Pensiones (1993-2003): avances, retos y reformas; Primera Edición. Editorial Universidad del Pacífico - Centro de Investigación. Lima 11 - Perú, http://www.slideshare.net/asociacionafp/historia-del-sistema-privado-de-pensiones-10-aos

46/ QVIPURAMAYOC | Vol. 21(39) 2013 
5. Javier Olivera $2013 \mathrm{El}$ objetivo olvidado: las pensiones, publicado el9 enero, 2013; en http://javierolivera.lamula.pe/2013/01/09/elobjetivo-olvidado-las-pensiones/emile

6. Héctor Cerna, Jaime Torres y Esteban Avelino. 2013 Cálculo de Reservas Actuariales en los Sis- temas de PensionesAdministradas por el Estado. Informe Final del Proyecto de Investigación, Sin - Sin, Instituto de Investigación de Ciencias Financieras y Contables de la Facultad de Ciencias Contables de la UNMSM. 